\title{
¿CUÁN INVOLUCRADO ESTOY? UN ESTUDIO EXPLORATORIO SOBRE EL COMPROMISO ACADÉMICO Y LA ORIENTACIÓN TEMPORAL EN ESTUDIANTES ARGENTINOS
}

\author{
HOW INVOLVED AM I? \\ AN EXPLORATORY STUDY ON ACADEMIC \\ ENGAGEMENT AND TEMPORARY \\ ORIENTATION IN ARGENTINE STUDENTS
}

Florencia Teresita Daura

Julio César Durand

PhD. en Ciencias de la Educación. Profesora Adjunta de la Escuela de Educación, Universidad Austral, Buenos Aires, Argentina. Investigadora Asistente del CIIPME (Centro Interdisciplinario de Investigaciones en Psicología Matemática y Experimental)-CONICET (Consejo Nacional de Investigaciones Científicas y Técnicas), Buenos Aires, Argentina.

fdaura@educ.austral.edu.ar

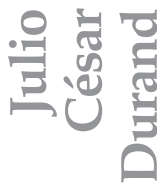

PhD. en Educación, Universidad de Navarra. Profesor Titular y Decano de la Escuela de Educación, Universidad Austral, Buenos Aires, Argentina.

jdurand@autral.edu.ar 


\section{RESUMEN}

Se presenta una investigación de carácter exploratoria y descriptiva efectuada en una muestra integrada por estudiantes argentinos de nivel medio, terciario y universitario con dos objetivos precisos: profundizar el estudio sobre el compromiso académico y la orientación temporal; y examinar la vinculación existente entre ambos constructos.

A tal fin, en primer lugar, se describen los fundamentos teóricos de la propuesta; a continuación, se detallan los instrumentos que se aplicaron, entre los que se destaca una escala diseñada ex profeso dirigida a evaluar el compromiso académico, y el Inventario de Perspectiva Temporal (Zimbardo y Boyd, 1999). Finalmente, se describen los resultados encontrados, que muestran correlaciones significativas entre las variables que integran los instrumentos seleccionados para validación, lo que permitirá utilizarlos en futuras investigaciones sobre el compromiso académico.

Palabras clave: compromiso académico, perspectiva temporal, asesoramiento académico, educación del carácter.

\section{ABSTRACT}

In this paper, an exploratory and descriptive research is presented to develop and increase the study on the relationship between academic engagement and temporal orientation. The study examines the link between both constructs in a sample of Argentine students at secondary, tertiary and university level.

First, the theoretical foundations of the proposal are described; then, the instruments that were applied - a scale specifically designed to assess the academic engagement, and the Temporal Perspective Inventory (Zimbardo \& Boyd, 1999) — are detailed. Lastly, the results are presented, showing significant correlations between the variables that make up the selected instruments, which are validated by this study for further research on academic engagement. 
Key words: academic engagement, temporal perspective, academic advice, character education.

\section{INTRODUCCIÓN}

En Latinoamérica y en la República Argentina, el estudio sobre el compromiso académico es incipiente, encontrándose publicaciones muy recientes focalizadas en el estudio del constructo en los primeros niveles del sistema educativo. Particularmente, se destacan los trabajos de Arguedas Negrini (2010), Dabenigno, Larripa, Austral, Tissera y Goldenstein Jalif (2010), Dabenigno y Tissera (2011), Dabenigno, Larripa y Austral (2012), Larripa (2012), Rigo (2013) y Rigo y Donolo (2014a, 2014b). En todas se destaca la utilización de metodología cualitativa, mixta o cuantitativa y, en estas últimas, el empleo de cuestionarios diseñados en forma ad hoc para el caso (Rigo, 2013).

Ya en anteriores publicaciones (Daura, 2015, 2016) se resalta el foco preventivo del constructo, explicando cómo su desarrollo puede incentivar la retención de los estudiantes dentro del sistema formal de enseñanza.

Asimismo, se señala (Daura, 2016) cómo el abordaje del compromiso académico, comenzó realizándose con un enfoque unidireccional, brindando importancia solamente a una de las variables que lo conforman - las estrategias cognitivas que utiliza el estudiante, por ejemplo-, sin considerar la influencia recíproca que los factores motivacionales, cognitivos y contextuales tienen entre sí, y que influyen positiva o negativamente en su desarrollo.

Ante esta situación, el enfoque sociocognitivo - uno de los modelos teóricos identificados en su estudio (Daura, 2015) —, es el que brinda elementos que permiten comprenderlo en forma integral y abarcativa. En efecto, desde ese enfoque se brinda un rol preponderante a la actividad del alumno, a las acciones que se efectúan desde la organización educativa, a fin de favorecer el proceso de enseñanza-aprendizaje y al interjuego de las variables contextuales y personales. De allí es que se afirma que se trata de un «meta-constructo», conformado por tres dimensiones en 
permanente interacción: compromiso comportamental, compromiso cognitivo y compromiso emocional (Fredricks, Blumenfeld y Paris, 2004, en Daura, 2016).

A partir de un breve marco teórico, el presente trabajo busca responder algunos interrogantes: ¿cómo evaluar el compromiso académico desde una visión sociocognitiva?, ¿en qué medida el compromiso académico de estudiantes de nivel medio y superior puede afectarse por la perspectiva temporal que posean?, ¿cómo emplear los datos recabados para desarrollar el carácter y la personalidad de los estudiantes?

\section{MARCO TEÓRICO DE REFERENCIA El Compromiso Académico}

El estudio del Compromiso Académico (también denominado involucración académica o, en inglés, engagement), tiene sus antecedentes en las investigaciones sobre la deserción y la retención estudiantil, en una línea que fue modificando el foco en el que se centraban los investigadores (Tinto, 2006-2007, en Daura, 2015). En concreto, comenzó a estudiarse a mediados de 1990, como consecuencia de las indagaciones que Astin (1984) efectuó en torno a la participación del estudiante en sus aprendizajes.

Si bien existen múltiples definiciones sobre el constructo, la que más se utiliza es la que brindan Kuh et al. (2008), quienes afirman que el compromiso estudiantil concierne, por un lado, al tiempo y las energías que los alumnos invierten para efectuar las actividades académicas -lo que es considerado también en los modelos de aprendizaje autorregulado- y, por el otro, al esfuerzo de las instituciones para implementar prácticas educativas efectivas. De esta manera, el concepto entrelaza las variables que forman parte de la dimensión subjetiva (entre ellas, la motivación, la cognición y el comportamiento) y las que conforman la dimensión contextual (que se concretan en el apoyo que la institución brinda al estudiante), que influyen en el proceso de aprendizaje y, consecuentemente, en la participación en la vida académica, la obtención de un buen rendimiento, el aumento de la satisfacción y la perseverancia frente al estudio (Daura, 2015). 
En un primer trabajo (Daura, 2015) se identificaron tres perspectivas teóricas: la constructivista, que asigna un papel fundamental al rol del estudiante (Krause y Coates, 2008); la sociocrítica, que postula que se puede desarrollar en forma participativa y dialógica (McMahon y Portelli, 2004); y la planteada por la teoría sociocognitiva la cual reconoce que la involucración está formada por cuatro tipos de compromiso: comportamental, emocional, cognitivo y contextual (Fredericks, Blumenfeld y Paris, 2004; Trowler, 2010).

A éstas se agrega la planteada por la Psicología Positiva (Seligman, 1999; Seligman y Csikszentmihalyi, 2000), que define el compromiso académico «un estado mental positivo relacionado con el trabajo, que se caracteriza por el vigor, la dedicación y la absorción» (Schaufeli et al., 2002, p. 79) y que también se aplica en relación con el desempeño académico.

De estos cuatro marcos conceptuales, el ofrecido por la teoría sociocrítica, es el que se considera apropiado para indagar el constructo en forma exhaustiva e integral, al dar importancia a la relación entre los aspectos intrapsíquicos —en particular la cognición y la motivación-y los contextuales: las acciones que promueve la institución para favorecer la involucración del estudiante. En efecto, su abordaje posibilita conocer en forma integrada qué acciones realizan los establecimientos educativos para favorecer el compromiso del estudiante y cuáles son las que éste efectúa para involucrarse más con su proceso de aprendizaje y con el contexto académico.

\section{Perspectiva Temporal}

El concepto de Perspectiva Temporal (PT) fue introducido por Lewin (1942), que lo comprende como una dimensión particular de la conducta humana (Daura, 2016). Entre los modelos que surgieron a partir del trabajo de Lewin y que se utiliza como referencia en este contexto, se destaca el elaborado por Zimbardo y Boyd (1999), en el que la PT se concibe como un proceso influido por las vivencias a las que se les asignan categorías temporales cognitivas que permiten ordenarlas con coherencia y brindarles un significado (Daura, 2016). Estos marcos cognitivos pueden ser modelos temporales repetidos o ser eventos únicos en la vida, que permiten codificar, almacenar y resignificar sucesos, metas y situaciones 
experimentadas en el momento de tomar una decisión y realizar una acción. La PT puede centrarse en el pasado, presente o futuro, puede aprenderse y modificarse, e influir sobre las acciones y decisiones que se adoptan durante el transcurso de la vida (Daura, 2016).

\section{METODOLOGÍA Y PROCEDIMIENTOS}

\section{Muestra}

Se conformó una muestra de 517 alumnos, de los cuales 176 se encontraban en el último año del nivel secundario y 350 cursaban el primer año de carreras terciarias y universitarias.

Con la muestra se efectuó una investigación de carácter exploratoria y descriptiva, ya que las variables a analizar se observaron tal y como se dan en su contexto natural en un momento dado.

\section{Objetivo}

Examinar la vinculación existente entre el compromiso académico y cinco factores temporales en estudiantes de nivel medio y superior.

\section{INSTRUMENTOS}

\section{Escala de Compromiso Académico}

Se trata de un instrumento diseñado en forma ex profesa, de acuerdo con los planteos de la teoría sociocognitiva (Fredricks y McColskey, 2012; Trowler, 2010). Posee un formato de escala tipo Likert compuesto por 56 ítems $^{1}$, con cinco opciones de respuesta (" 0 ", que significa "completamente erróneo", "1" "erróneo", "2" "ni verdadero ni erróneo", "3" "verdadero" y "4" "completamente verdadero").

1 Ejemplos de ítems: «19. En mi facultad se ofrecen diversas oportunidades de participación social». «40. Sería muy comprometedor y motivante que un profesor me convoque para formar parte de una cátedra». «53. En la facultad se brindan orientaciones para adquirir un empleo o competencias laborales». 


\section{Inventario de Perspectiva Temporal (Zimbardo Time Perspective Inventory, ZTPI)}

El Inventario (Zimbardo y Boyd, 1999), cuya validez y confiabilidad se demostró en numerosas investigaciones, tiene un formato de escala de respuestas tipo Likert (con enunciados positivos y negativos, para evitar la respuesta mecánica). Posee cinco alternativas de respuesta y, al igual que el anterior instrumento, cuenta con 56 ítems que exploran cinco factores temporales claramente identificados: Pasado Negativo $(\mathrm{PN})$, expresa el rechazo o la visión pesimista que se tiene sobre la vida pasada; Pasado Positivo (PP), evalúa los sentimientos positivos y de nostalgia hacia las experiencias vividas; Presente Hedonista $(\mathrm{PH})$, evalúa la orientación a buscar el placer en el momento presente, sin reflexionar en demasía sobre las consecuencias que ello tendrá en el futuro; Presente Fatalista $(\mathrm{PF})$, refleja una actitud de indefensión, desesperanza y resignación frente a la vida y al futuro; y Futuro (F), expresa cómo el sujeto procura alcanzar objetivos y recompensas futuras.

\section{Procedimiento}

Se solicitó el permiso correspondiente a las autoridades de las instituciones que participaron en el estudio; de la misma manera, a los alumnos que completaron los cuestionarios, se les brindó información sobre los objetivos del trabajo, el carácter confidencial y voluntario de su participación, y se les entregó un documento para dar su consentimiento.

El tiempo empleado para completar los instrumentos varió entre 40 y 60 minutos.

Los puntajes de la Escala de de Compromiso Académico se obtuvieron sumando las elecciones de los sujetos y convirtiendo la suma a escala $10^{2}$. Los del Inventario de Perspectiva Temporal se convirtieron a escala 5.

Los datos fueron procesados mediante la utilización del programa SPSS (Statistical Package for the Social Sciences) ${ }^{3}$, versión 23.0.

2 El puntaje máximo posible se dividió por la cantidad de ítems de la variable y se lo multiplicó por 10.

3 Programa estadístico para las Ciencias Sociales. 


\section{ANÁLISIS DE LOS RESULTADOS}

\section{Estructura factorial de la Escala de Compromiso Académico (EsCA):}

Se efectuó un análisis factorial exploratorio con rotación Varimax, a fin de evaluar la validez de constructo del cuestionario. Se obtuvo el índice de adecuación de la muestra de Keiser-Meyer-Olkin (KMO), que arrojó un coeficiente de 0,901 y la prueba de esfericidad de Barlett que evidenció una significación $\mathrm{p}=0,0001$ y una varianza total explicada de $59,25 \%$, resultados que indican que la matriz de datos es adecuada para realizar el análisis.

Fruto de este análisis, se extrajeron trece variables distribuidas en tres subescalas, que se denominaron según el detalle de la tabla 1. 


\section{Tabla 1. Descripción de las 13 subescalas extraídas}

\begin{tabular}{|c|c|}
\hline \multicolumn{2}{|c|}{$\begin{array}{l}\text { Subescala 1. Compromiso Motivacional: conformada por } 17 \text { ítems, explora cuatro componentes afectivo- } \\
\text { motivacionales: }\end{array}$} \\
\hline $\begin{array}{l}\text { Valoración de la } \\
\text { tarea }\end{array}$ & $\begin{array}{l}\text { Refleja la utilidad, la importancia y el valor otorgado a los contenidos, a las } \\
\text { actividades de aprendizaje que se reciben en la institución. }\end{array}$ \\
\hline Motivación intrínseca & $\begin{array}{l}\text { Expresa el interés que se posee por aprender los contenidos y/o el material } \\
\text { bibliográfico que se propone desde la institución. }\end{array}$ \\
\hline $\begin{array}{l}\text { Motiva } \\
\text { extríns }\end{array}$ & $\begin{array}{l}\text { Demuestra el interés por estudiar con el fin de satisfacer motivos externos } \\
\text { (como por ejemplo obtener buenas notas, recibir el reconocimiento ajeno), y de } \\
\text { aprender apoyándose en la guía que ofrece el contexto. }\end{array}$ \\
\hline nsie & $\begin{array}{l}\text { Refleja la ansiedad que se experimenta ante situaciones estresantes de la vida } \\
\text { académica, como puede ser un examen. }\end{array}$ \\
\hline \multicolumn{2}{|c|}{$\begin{array}{l}\text { Subescala 2. Compromiso Cognitivo: formada por } 27 \text { ítems que exploran las estrategias cognitivas que se } \\
\text { utilizan para aprender, a través de las siguientes variables: }\end{array}$} \\
\hline Estrategias profundas & $\begin{array}{l}\text { Refleja en qué medida se emplean estrategias de elaboración y el grado con el } \\
\text { cual se utilizan conocimientos previos para resolver problemas, tomar decisiones, } \\
\text { realizar evaluaciones críticas, aceptar las opiniones ajenas. }\end{array}$ \\
\hline Estrat & $\begin{array}{l}\text { de repaso para aprender a } \\
\text { tos claves. }\end{array}$ \\
\hline Organ & s importantes del \\
\hline $\begin{array}{l}\text { Regul } \\
\text { extrín }\end{array}$ & $\begin{array}{l}\text { trategias que aplica el sujeto cuando se ajusta a las indicaciones } \\
\text { el docente. }\end{array}$ \\
\hline $\begin{array}{l}\text { Gestión } \\
\text { del esfu }\end{array}$ & $\begin{array}{l}\text { osición personal para esforzarse frente al trabajo } \\
\text { sea difícil-y la capacidad para organizar el tiempo }\end{array}$ \\
\hline Trabajo en equipo & Refleja la disposición para trabajary aprender en equipo, en el ámbito institucional. \\
\hline \multicolumn{2}{|c|}{$\begin{array}{l}\text { Subescala 3. Compromiso Contextual: formada por } 12 \text { ítems que evalúan tanto las acciones que la } \\
\text { institución realiza para apoyar al estudiante y promover su involucración académica, así como también } \\
\text { la participación de este último en las actividades propuestas por la institución y su identificación con los } \\
\text { valores promovidos por la organización educativa. Los ítems se distribuyen en las siguientes variables: }\end{array}$} \\
\hline $\begin{array}{l}\text { Valoración positiva } \\
\text { de la institución }\end{array}$ & $\begin{array}{l}\text { Indica el grado con el cual el estudiante valora diversas acciones que se } \\
\text { realizan desde la institución para promover distintas competencias y propiciar la } \\
\text { integración entre los alumnos. }\end{array}$ \\
\hline Tutoría y & $\begin{array}{l}\text { Refleja en qué medida se valoran las acciones que realizan los tutores y los } \\
\text { docentes para facilitar el aprendizaje de los alumnos y favorecer su participación } \\
\text { en la institución. }\end{array}$ \\
\hline $\begin{array}{l}\text { Sentimientos de } \\
\text { ertenencia }\end{array}$ & $\begin{array}{l}\text { Evalúa los sentimientos de pertenencia que tiene el alumno y que son } \\
\text { promovidos por la institución a través de distintas actividades académicas. }\end{array}$ \\
\hline
\end{tabular}

Fuente: Elaboración propia. 


\section{Análisis de confiabilidad por variables y subescalas que conforman el instrumento}

A fin de ponderar la confiabilidad del instrumento, se empleó el alpha de Cronbach (tabla 2), que alcanzó coeficientes que oscilan entre 0,90 y 0,38 . Particularmente, por tratarse de los más elevados, se destacan los valores alcanzados por el instrumento en su totalidad $(0,81)$ y por las variables "Valoración de la Tarea" $(0,90)$ y "Tutoría y Docencia" $(0,83)$. Contrariamente, la variable "Ansiedad" fue la que alcanzó la menor puntuación $(0,38)$, posiblemente por la cantidad de ítems que la integran (Nunnally, 1967).

Por tratarse de un estudio de carácter exploratorio, estos resultados, de acuerdo con Nunnally (1978), son fiables y suficientes.

Tabla 2. Resultados del Análisis de Confiabilidad

\begin{tabular}{|l|c|}
\hline Subescala Compromiso Motivacional & 0,62 \\
\hline Valoración de la tarea & 0,90 \\
\hline Motivación intrínseca & 0,72 \\
\hline Motivación extrínseca & 0,53 \\
\hline Ansiedad & 0,38 \\
\hline Subescala Compromiso Cognitivo & 0,52 \\
\hline Estrategias profundas & 0,77 \\
\hline Estrategias básicas & 0,76 \\
\hline Organización & 0,70 \\
\hline Regulación extrínseca & 0,66 \\
\hline Gestión del tiempo y del esfuerzo & 0,70 \\
\hline Trabajo en equipo & 0,74 \\
\hline Subescala Compromiso Contextual & 0,69 \\
\hline Valoración positiva de la institución & 0,76 \\
\hline Tutoría y docencia & 0,83 \\
\hline Sentimientos de pertenencia & 0,72 \\
\hline Escala total & 0,81 \\
\hline
\end{tabular}

Fuente: Elaboración propia. 


\section{Análisis descriptivo de la Escala de Compromiso Académico}

Se realizó un análisis descriptivo (tabla 3) cuyos resultados expresan que la muestra posee un compromiso motivacional, caracterizado por la valoración de los contenidos y de las actividades de aprendizaje propuestas en la institución, junto con un interés manifiesto por aprehenderlos e involucrarse en ellos y un índice elevado de ansiedad; esto último, podría vincularse al momento de la carrera en el que se encuentran los estudiantes, que en su mayoría están iniciando en la vida universitaria o finalizando el nivel secundario, situación que comúnmente genera incertidumbre, necesidad de adaptarse a un ambiente nuevo y desconocido, así como también a la exigencia de tomar decisiones esenciales en el proyecto vital de toda persona. En lo que respecta al compromiso cognitivo, los sujetos se destacan por poseer la capacidad para organizar el material de estudio, elaborar un pensamiento crítico y por considerar las orientaciones brindadas por los docentes para estudiar.

En cuanto al compromiso contextual, los alumnos se caracterizan por poseer una valoración positiva de la institución de la que forman parte y de tener sentimientos de pertenencia hacia ésta, resultados que se vinculan entre sí y se destacan si se considera que los sujetos pertenecían a organizaciones educativas muy diversas.

Del mismo modo, estos puntajes mostrarían una cualidad a desatacar en los estudiantes que participaron en el estudio, que está en línea con el «modelo contextual del compromiso académico» desarrollado por Lam, Wong, Yang y Liu (2012), en el que se explica que el aumento de la involucración hacia la institución favorece el desarrollo de sentimientos positivos hacia el contexto de aprendizaje, hacia las demás personas con las que interactúan en este ambiente y hacia la propia capacidad para aprender. En efecto, este tipo de compromiso propicia el aumento de la motivación intrínseca, de la valoración de la tarea, de la autoconfianza para enfrentar desafíos académicos y fortalece los lazos que se entablan con compañeros de clase, profesores, directivos y asesores (Daura, 2016). 
Tabla 3. Análisis descriptivo de las variables que integran la escala de Compromiso Académico

\begin{tabular}{|l|r|r|r|r|r|}
\hline \multicolumn{1}{|c|}{ Variable } & \multicolumn{1}{|c|}{ N } & Mínimo & Máximo & Media & $\begin{array}{c}\text { Desv. } \\
\text { típ. }\end{array}$ \\
\hline Valoración de la tarea & 517 &, 94 & 10,00 & 6,47 & 1,98 \\
\hline Motivación intrínseca & 517 & 0,00 & 10,00 & 6,41 & 2,00 \\
\hline Motivación extrínseca & 517 & 0,00 & 10,00 & 5,84 & 2,11 \\
\hline Ansiedad & 517 & 0,00 & 10,00 & 4,40 & 2,28 \\
\hline Estrategias profundas & 517 &, 42 & 10,00 & 5,70 & 1,74 \\
\hline Estrategias básicas & 517 & 0,00 & 10,00 & 4,58 & 2,31 \\
\hline Organización & 517 & 0,00 & 10,00 & 6,90 & 2,00 \\
\hline Regulación extrínseca & 517 & 0,00 & 10,00 & 6,21 & 1,80 \\
\hline $\begin{array}{l}\text { Gestión del tiempo y del } \\
\text { esfuerzo }\end{array}$ & 517 &, 50 & 10,00 & 5,46 & 1,85 \\
\hline Trabajo en equipo & 517 &, 83 & 10,00 & 5,81 & 1,72 \\
\hline $\begin{array}{l}\text { Valoración positiva de la } \\
\text { institución }\end{array}$ & 517 & 0,00 & 10,00 & 6,61 & 2,02 \\
\hline Tutoría y docencia & 517 & 0,00 & 10,00 & 4,59 & 2,39 \\
\hline Sentimientos de pertenencia & 517 & 0,00 & 10,00 & 5,84 & 2,12 \\
\hline
\end{tabular}

Fuente: Elaboración propia.

\section{Análisis descriptivo del Inventario de Perspectiva Temporal}

En este caso, también se calcularon los valores medios y el desvío estándar de los factores que conforman el cuestionario de Zimbardo y Boyd (1999) (tabla 4). A partir de los resultados obtenidos, los estudiantes evidencian poseer una perspectiva temporal equilibrada, caracterizada por la visión optimista del pasado, la búsqueda de la satisfacción en el tiempo un presente y la posibilidad de fijar metas posibles de ser alcanzadas en el futuro. 
Tabla 4. Valores descriptivos del inventario de Perspectiva Temporal

\begin{tabular}{|l|c|c|c|r|r|}
\hline & N & Mínimo & Máximo & Media & \multicolumn{1}{c|}{ Desv. típ. } \\
\hline Pasado negativo & 517 & 1,00 & 4,70 & 2,92 & 0,67 \\
\hline Pasado positivo & 517 & 2,13 & 4,88 & 3,61 & 0,49 \\
\hline Presente hedonista & 517 & 1,27 & 5,00 & 3,37 & 0,55 \\
\hline Presente fatalista & 517 & 1,00 & 4,33 & 2,60 & 0,60 \\
\hline Futuro & 517 & 1,60 & 5,00 & 3,48 & 0,53 \\
\hline
\end{tabular}

Fuente: Elaboración propia.

\section{CORRELACIÓN ENTRE LAS VARIABLES QUE INTEGRAN LA ESCALA DE COMPROMISO ACADÉMICO Y LOS FACTORES TEMPORALES}

A fin de comprobar la vinculación existente entre las variables que integran la Escala de Compromiso Académico y los factores temporales, se calcularon los índices de correlación $r$ de Pearson: 


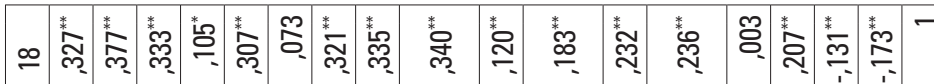

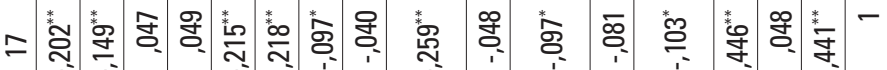

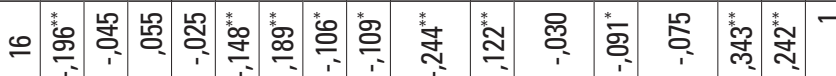

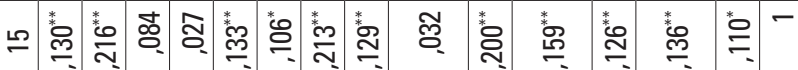

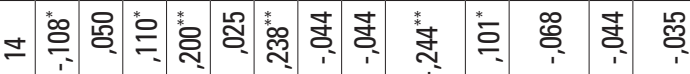

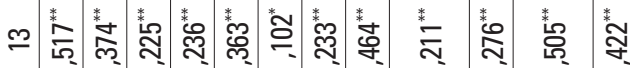

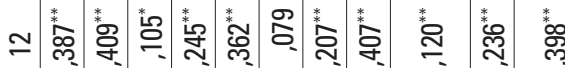

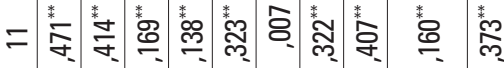

동

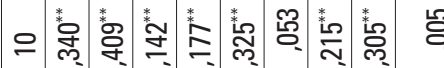

응 는

$\frac{\pi}{6}$

( $E$

ס

(1)

थ

त्व

ن

(1)

윽 으

๘ $\frac{\bar{\sigma}}{2}$

즈 ह

인

둥

(2)

ฮั ฮ

प

점 잉

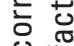

(1) ल

응 으

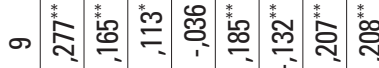

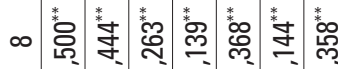

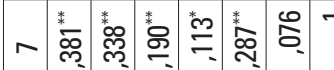

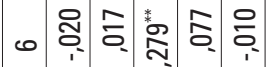

น

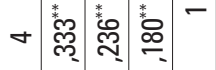

๓

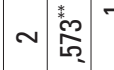

Nㅏㄷ

$\sum^{\infty} \cdot \frac{\omega}{E}$

เก 잉

드을

ह

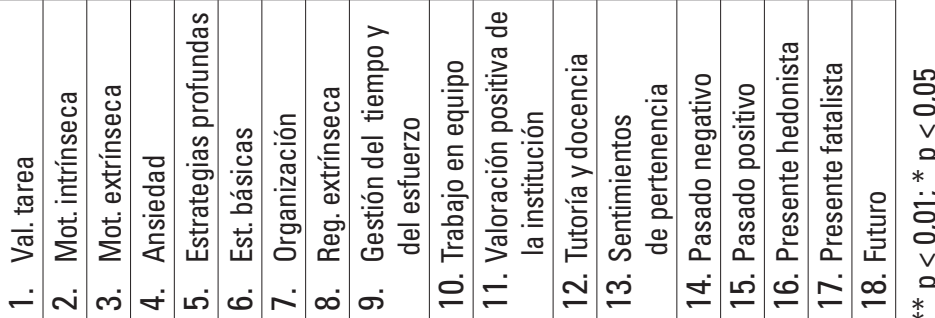


Fruto de este análisis, destacan las correlaciones positivas y significativas halladas entre las variables que integran la subescala de Compromiso Motivacional ${ }^{4}$, y entre éstas y las que conforman las subescalas de Compromiso Cognitivo ${ }^{5}$ y de Compromiso Contextual ${ }^{6}$. Lo que coincide con las ideas expuestas en estudios anteriores: en la medida en que se alcanzan mayores índices motivacionales, aumenta la involucración cognitiva y contextual (Fredricks, Blumenfeld y Paris, 2004).

Igualmente, se encontró este tipo de correlación entre las variables de la subescala de Compromiso Motivacional y algunos de los factores temporales; en concreto entre Valoración de la Tarea y Pasado Positivo $\left[r=(517)=0,517^{* *}, p=0,01\right]$ y Presente Hedonista $\left[r=(517)=0,130^{* *}, p=\right.$ $0,01]$ y Futuro $\left[r=(517)=0,327^{\star *}, p=0,01\right]$. También entre Metas Intrínsecas y Presente Positivo $\left[r=(517)=0,216^{* *}, p=0,01\right]$ y Futuro $\left[r=(517)=0,377^{* *}\right.$, $p=0,01]$. Estos resultados reflejan que el valor brindado al aprendizaje de nuevos contenidos y al interés por aprehenderlos se asocian a la adquisición de una perspectiva temporal equilibrada.

En el caso de Metas Extrínsecas, la correlación se presentó con los factores Pasado Negativo $\left[r=(517)=0,110^{*}, p=0,05\right]$ y Futuro $[r=(517)=$ $\left.0,333^{* *}, p=0,01\right]$; en los que podría encontrarse una asociación entre las vivencias desagradables y la necesidad de recibir un estímulo externo para obtener un beneficio compensatorio o evitar la repetición de estos sucesos.

4 Por ejemplo, entre la variable Valoración de la Tarea y Metas Intrínsecas $\left[r=(517)=0,573^{* *}\right.$, $p=0,01]$; entre Metas Intrínsecas y Metas Extrínsecas $\left[r=(517)=0,227^{* *}, p=0,01\right]$, efecto que mostraría la vinculación entre ambos tipos de metas, siendo necesarias para favorecer el aumento de la valoración de la tarea (Pintrich, 2000).

5 Por ejemplo, entre Valoración de la Tarea y Estrategias Profundas $\left[r=(517)=0,580^{\star *}, p=0,01\right]$; entre Valoración de la Tarea y Organización $\left[r=(517)=0,381^{* *}, p=0,01\right]$; entre Valoración de la Tarea y Regulación Extrínseca $\left[r=(517)=0,500^{* *}, p=0,01\right]$; entre Valoración de la Tarea y Gestión del Tiempo y del Esfuerzo $\left[r=(517)=0,277^{* *}, p=0,01\right]$; entre Valoración de la Tarea y Trabajo en Equipo $\left[r=(517)=0,340^{* *}, p=0,01\right]$.

Entre Metas Intrínsecas y Estrategias Profundas $\left[r=(517)=0,563^{* *}, p=0,01\right]$; entre Metas Intrínsecas y Organización [ $\left.r=(517)=0,338^{* *}, p=0,01\right]$; entre Metas Intrínsecas y Regulación Extrínseca $\left[r=(517)=0,444^{* *}, p=0,01\right]$; entre Metas Intrínsecas y Gestión del Tiempo y del Esfuerzo $\left[r=(517)=0,165^{* *}, p=0,01\right]$; por mencionar sólo algunas.

6 Algunos ejemplos se encuentran entre Motivación Extrínseca y Valoración Positiva de la Institución $\left[r=(517)=0,169^{* *}, p=0,01\right]$; Tutoría y Docencia $\left[r=(517)=0,105^{*}, p=0,05\right]$; y Sentimientos de Pertenencia $\left[r=(517)=0,225^{* *}, p=0,01\right]$. 
El mismo efecto se encontró entre la variable Ansiedad y el Pasado Negativo $\left[r=(517)=0,200^{* *}, p=0,01\right]$ y el Futuro $\left[r=(517)=0,105^{*}, p=\right.$ $0,05]$, con lo cual, el manejo de las situaciones estresantes estaría influido negativamente por las experiencias desagradables que se hayan vivido, pero podría regularse adecuadamente en el caso de saberse capaz para elegir objetivos vitales y de tener la autoconfianza para alcanzarlos en el futuro.

En lo que respecta a la subescala de Compromiso Cognitivo, se encontraron correlaciones positivas y significativas entre las variables Estrategias Profundas y Organización $\left[r=(517)=0,287^{*}, p=0,05\right]$, lo que evidencia la necesidad de ordenar los conocimientos para luego desarrollar un pensamiento crítico y elaborado (Marton y Saljo, 1976). Asimismo, las Estrategias Profundas correlacionaron en forma positiva y significativa con las Estrategias Básicas, efecto que desmitifica la irrelevancia de la repetición necesaria para aprender; efectivamente, para aprender determinados contenidos se requiere de la memorización, para luego establecer vinculaciones entre ellos y desarrollar un juicio propio al respecto.

Las demás correlaciones encontradas entre la variable Estrategias Profundas y otras variables de la misma subescala ${ }^{7}$, como con aquellas que integran las subescalas Compromiso Motivacional y Compromiso Contextual $^{8}$, se asocian a la idea que varios autores señalan: la obtención de mayores niveles de compromiso académico, está ligada al desarrollo de un aprendizaje profundo (Fredericks, Blumenfeld y Paris, 2004; Yorke, 2006).

Igualmente, se encontraron correlaciones positivas y significativas entre algunas de las variables que integran la subescala Compromiso Cognitivo con el factor Pasado Positivo y con el factor Futuro, lo que permitiría asociar mayores niveles de involucración cognoscitiva con la visión positiva del pasado $\mathrm{y}^{9} \mathrm{y}$ de un futuro próspero ${ }^{10}$.

\footnotetext{
Por ejemplo, entre Estrategias Profundas y Organización $\left[r=(517)=0,287^{* *}, p=0,01\right]$.

8 Estrategias Profundas y Valoración Positiva de la Institución $\left[r=(517)=0,323^{\star *}, p=0,01\right]$.

9 Ello se ve en la correlación positiva y significativa entre las siguientes variables con el factor Pasado Positivo: Estrategias Profundas $\left[r=(517)=0,133^{* *}, p=0,01\right]$; Estrategias Básicas $\left[r=(517)=0,106^{*}, p=0,05\right]$; Organización $\left[r=(517)=0,213^{* *}, p=0,01\right]$; Regulación Extrínseca $\left[r=(517)=0,129^{* *}, p=0,01\right]$ y Trabajo en Equipo $\left[r=(517)=0,200^{* *}, p=0,01\right]$.

10 La correlación aludida se encuentra entre todas las variables de la subescala y el factor futuro, con excepción de Estrategias Básicas.
} 
Particularmente, se destaca la asociación entre la variable Manejo del Tiempo y del Esfuerzo con el factor Futuro $\left[r=(517)=0,340^{* *}, p=0,01\right]$, que expresa que la capacidad para regular el espacio y la agenda personal de estudio estaría en vinculación con la visión que se tiene sobre el porvenir. Es decir, en cuanto a éste se lo percibe como positivo y, simultáneamente, factible de ser gobernado, la persona se organizaría mejor para estudiar, priorizar y afanarse para asimilar conocimientos.

En cuanto a la subescala Compromiso Contextual, las variables que la integran -más allá de las correlaciones positivas encontradas con las demás variables del EsCA-, correlacionaron positiva y significativamente con los factores Pasado Positivo y Futuro.

Las correlaciones negativas y significativas entre estas variables y el Pasado Negativo, el Presente Hedonista y el Presente Fatalista, mostrarían que la influencia favorable del entorno podría compensar las experiencias ingratas del pasado, contrarrestar la búsqueda de las satisfacciones inmediatas y la concepción fatídica del presente.

Esto marca la importancia del rol del docente y del tutor en el proceso educativo a fin de incentivar el aprendizaje de los alumnos, promover su participación e involucración en las actividades propuestas y evitar el abandono en sus estudios.

\section{CONCLUSIONES}

Los constructos abordados en el presente trabajo tienen gran vigencia en todos los niveles educativos. En concreto, el compromiso académico se concibe como un proceso en el cual se fortalece el vínculo entre el estudiante y la institución educativa, a partir de la influencia que esta última ejerce para promover que el primero tenga un mayor sentimiento de pertenencia y que finalice sus estudios (Daura, 2015, 2016).

La Perspectiva Temporal es «un proceso no consciente, mediante el cual el flujo de las experiencias personales y sociales se encuadran en categorías temporales que ayudan a dar orden, coherencia y significado a esas experiencias» (Zimbardo y Boyd, 1999, p. 1271). 
A partir de la aplicación de una escala de compromiso académico, diseñada en forma ex profeso y del Inventario de Perspectiva Temporal (Zimbardo y Boyd, 1999), se puso a prueba la vinculación que guardan ambos constructos en estudiantes argentinos de nivel medio, terciario y universitario.

El análisis de la estructura factorial de la Escala de Compromiso Académico permitió extraer trece variables distribuidas en tres subescalas claramente identificadas y definidas a nivel teórico. La primera de ellas, a la que se denomina Subescala de Compromiso Motivacional, explora cuatro componentes afectivo-motivacionales que inciden en el aumento o en la disminución de la involucración académica: Valoración de la Tarea, Motivación Intrínseca, Motivación Extrínseca y Ansiedad.

La segunda, denominada Compromiso Cognitivo, explora las estrategias cognitivas que se utilizan para aprender y que inciden en la involucración del estudiante hacia su estudio y en el vínculo que mantiene con la institución de enseñanza: Estrategias Profundas, Estrategias Básicas, Organización, Regulación Extrínseca, y Gestión del Tiempo y del Esfuerzo, Trabajo en Equipo.

La tercera, Compromiso Contextual, está formada por 12 ítems que se distribuyen en 3 variables que evalúan tanto las acciones que la institución realiza para apoyar al estudiante y promover su involucración, así como también la participación de este último en las actividades propuestas y su identificación con los valores promovidos por la organización educativa.

Para analizar la confiabilidad del instrumento, se empleó el alpha de Cronbach que arrojó coeficientes que oscilan entre 0,90 y 0,30 que, de acuerdo con Nunnally (1978), son fiables y suficientes por tratarse de un estudio de carácter exploratorio.

La correlación entre los instrumentos seleccionados arrojó resultados que muestran la existencia de correlaciones positivas y significativas entre la mayoría de las variables que conforman las tres subescalas del EsCA entre sí, lo que marca la vigencia del enfoque sociocognitivo para explicar el constructo. En efecto, cuanto mayor sea el empleo de estrategias motivacionales y cognitivas, y cuanto mayor sea la involucración con el contexto, mejores niveles de involucración se tendrán con el propio 
aprendizaje y con el entorno institucional que rodea al estudiante. Claro está que, en el interjuego de estas variables, no sólo tienen peso las acciones del estudiante, sino también las que se desenvuelven en el entorno y, en forma particular, las de los profesores, tutores y directivos, y hasta de los demás alumnos que integran la organización educativa.

Del mismo modo, se hallaron correlaciones significativas y positivas entre las subescalas del EsCA y los factores Pasado Positivo, Presente Hedonista y Futuro, lo que mostraría que los niveles de compromiso académico aumentan en la medida en que se tenga una perspectiva temporal equilibrada, caracterizada por el recuerdo positivo del pasado, la vivencia de un presente reconfortante y la visión de un futuro próspero y deseable.

Los resultados recabados, que se relacionan con los trabajos de Horstmanshof y Zimitat (2007), y Daura (2016) por un lado, permiten corroborar la vinculación existente entre el compromiso académico y la perspectiva temporal, y por el otro, se constituyen en el segundo estudio realizado al respecto en la población argentina.

Las relaciones encontradas entre las variables que integran el compromiso académico y los factores temporales, permiten considerar el efecto que el entorno ejerce en el desarrollo de una mayor involucración y de la consolidación de un proyecto vital por parte de los estudiantes, en el que ocupan un lugar preponderante las metas académicas y profesionales.

En efecto, si ambos constructos pueden modificarse por la influencia del proceso de enseñanza formal, suponen el desarrollo de hábitos o de acciones que por su ejercicio libre, racional y sentido se afianzan en el sujeto, configuran su forma de ser y le imprimen un carácter que forma parte de su personalidad.

Convendrá entonces atender cómo, desde el espacio del aula y de la tutoría personal o grupal, pueden generarse estrategias que favorezcan la promoción de estudiantes capaces de proyectarse en el tiempo, afianzar su proyecto de vida y comprometerse en alcanzar las metas de distinto tipo que se hayan propuesto y que favorezcan su crecimiento integral. 
En la sociedad actual, caracterizada por la «liquidez» (Bauman, 1999), la interrupción, la sorpresa y los cambios abruptos, inclusive para mantener ideales existenciales que en algún momento se consideraron como tales, el mantenimiento de los compromisos asumidos, supone ir más allá de su cumplimiento. En el plano de un proyecto académico, presupone valorar lo que se aprehende, valorar en dónde se aprende y autovalorar la propia capacidad para aprender; implica también juzgar críticamente los conocimientos adquiridos, compartirlos con respeto con otros compañeros y docentes; conlleva estar dispuesto a buscar ayuda cuando se presentan dificultades para afianzarlos; manifestar interés por participar en las actividades propuestas y por enriquecer la institución de la que se forma parte.

De acuerdo con estos planteos, el fomento del compromiso académico y de una perspectiva temporal equilibrada, obliga volver a las raíces de la educación, en pos de desarrollar integralmente la personalidad de cada educando.

\section{REFERENCIAS}

Arguedas Negrini, I. (2010). Involucramiento de las estudiantes y los estudiantes en el proceso educativo. Revista Iberoamericana sobre Calidad, Eficacia y Cambio en Educación, 8(1), 63-78

Astin, A. W. (1984). Student involvement: A developmental theory for higher education. Journal of College Student Development, 25, 297-308.

Bauman, Z. (1999). Modernidad líquida. México: Fondo de Cultura Económica.

Dabenigno, V. y Tissera, S. (2011). La escuela es un buen lugar. El involucramiento social y afectivo de los estudiantes. Lainvestigación en diálogo con la escuela $N^{o} 3$ del Boletín de la Educación Porteña $N^{o} 11$, Ministerio de Educación del GCBA. Consultado en https://goo. $\mathrm{gl} / \mathrm{DRGhZv}$ 
Dabenigno, V.; Larripa, S. y Austral, R. (diciembre, 2012). Inclusión educativa e involucramiento escolar. Perspectivas y prácticas en cuatro escuelas estatales de la Ciudad de Buenos Aires. Ponencia presentada en las VII Jornadas de Sociología de la Universidad Nacional de La Plata «Argentina en el escenario latinoamericano actual: debates desde las ciencias sociales». La Plata, Buenos Aires, Argentina. Consultado en https://goo.gl/jQfnsg

Dabenigno, V.; Larripa, S. y Austral, R. (diciembre, 2012). Inclusión educativa e involucramiento escolar. Perspectivas y prácticas en cuatro escuelas estatales de la Ciudad de Buenos Aires. Ponencia presentada en las VII Jornadas de Sociología de la Universidad Nacional de La Plata «Argentina en el escenario latinoamericano actual: debates desde las ciencias sociales». La Plata, Buenos Aires, Argentina.

Dabenigno, V.; Larripa, S.; Austral, R.; Tissera, S. y Goldenstein Jalif, Y. (2010). Permanencia e involucramiento escolar de los estudiantes secundarios. Perspectivas y acciones en cuatro escuelas estatales de la Ciudad de Buenos Aires. Dirección de Investigación y Estadística del Ministerio de Educación del Si, EG.C.B.A. Consultado en https://goo.gl/pVeNMS

Daura, F. T. (2015). El estudio del compromiso académico. Panorama general sobre su abordaje. Diálogos Pedagógicos, 13(25), 54-75.

Daura, F. T. (2016). El compromiso emocional de los estudiantes hacia el contexto educativo. Su vinculación con factores temporales y con variables demográficas. Revista de Orientación Educacional, 31(58), 54-73.

Fredricks, J. A.; Blumenfeld, P. C. \& Paris, A. H. (2004). School Engagement: Potential of the Concept, State of the Evidence. Review of Educational Research, 74(1), 59-109.

Fredricks, J. A. \& McColskey, W. (2012). The Measurement of Student Engagement: AComparative Analysis of Various Methods and Student Self-report Instruments. In: S. L. Christenson; A. L. Reschly y C. Wylie (Eds), Handbook of research on student engagement (pp. 763-782). New York, United States of America: Springer. 
Fredericks, J.A.; Blumenfeld, P.C. \& Paris, A.H. (2004). School Engagement: Potential of the Concept, State of the Evidence. Review of Educational Research, 74(1), 59-109.

Horstmanshof, L. \& Zimitat, C. (2007). Future time orientation predicts academic engagement among first-year university students, British Journal of Eductaional Psychology, 77, 703-718.

Krause, K. L. \& Coates, H. (2008). Students' Engagement in Firstyear University. Assessment y Evaluation in Higher Education, 33(5), 493-505.

Kuh, G. D; Cruce, T.; Shoup, R. y Kinzie, J. \& Gonyea, R. M. (2008). Unmasking the Effects of Student Engagement on First Year College Grades and Persistence. Journal of Higher Education, 79(5), 540-563.

Lam, S.; Wong, B.; Yang, H. \& Liu, Y. (2012). Understanding student engagement with a contextual model. In S. Christenson; A. Reschly y C. Wylie (Eds.), Handbook Research on Student Engagement (pp. 403-419). New York, United States of America: Springer.

Larripa, S. (2012). Trabajo e involucramiento docente en escuelas secundarias con proyectos de inclusión. La investigación en diálogo con la escuela $N^{o} 3$ del Boletín de la Educación Porteña $N^{o} 15$, Ministerio de Educación del GCBA.

Lewin, K. (1935). A dinamic theory of personality. New York, United States of America: Mc Graw-Hill.

McMahon, B. \& Portelli, J. (2004). Engagement for what? Beyond popular discourses of student engagement. Leadership and Policy in Schools, 3(1), 59-76.

Marton, F. \& Säljö, R. (1976). On qualitative differences in learning: I-outcome and process. British Journal of Educational Psychology, 46, 4-11.

Nunnally, J. C. (1978). Psychometric theory. New York, United States of America: McGraw Hill. 
Rigo, D. Y. (2013). Compromiso hacia las tareas académicas: diseños instructivos e inteligencias múltiples. Ikastorratza, 10, 1-15. Consultado en https:/ / goo.gl/DQvBEB

Rigo, D. Y. y Donolo, D. (2014a). Entre pupitres y pizarrones. Retos en educación primaria: el aprendizaje con compromiso. Educatio Siglo XXI, 32(2). 59-80

Rigo, D. Y. y Donolo, D. (2014b, noviembre). Factores clave en la promoción del compromiso en educación primaria. Un estudio sobre tasks, teachers and students. Trabajo presentado en el Congreso Iberoamericano de Ciencia, Tecnología, Innovación y Educación. Buenos Aires, Argentina. Artículo 326.

Seligman, M.E.P. (1999). The presidents address. APA.1998. Annual Report. American Psychologist, 54, 559-562.

Seligman, M. \& Csikszentmihalyi, M. (2000). Positive Psychology: An introduction. American Psychologist, 55(1), 5-14.

Schaufeli, W. B., Salanova, M., González-Romá, V. \& Bakker, A. (2002). The measurement of burnout and engagement: A confirmatory factor analytic approach. Journal of Happiness Studies, 3, 71-92.

Tinto, V. (2006-2007). Research and Practice of Student Retention: What next? College Student Retention, 8(1), 1-19.

Yorke, M. (2006). Student engagement: Deep, surface or strategic? Keynote address delivered at the 9th Pacific Rim First Year in Higher Education Conference: Engaging Students. Griffith University, Gold Coast, Australia, 12-14 July.

Zimbardo, P. G. \& Boyd, J. N. (1999). Putting time in perspective: A valid, reliable individual differences metric. Journal of Personality and Social Psychology, 77, 1271-1288. 